\title{
Hartree-Fock Equation for a Non-neutral Plasma of Spin Zero lons in a Paul Trap
}

\author{
Fernand Tshizanga Mpinga \\ Department of Mechanics, Superior Institute of Applied Techniques, Kinshasa, Democratic Republic of Congo
}

Email address:

tshizafer@hotmail.com

To cite this article:

Fernand Tshizanga Mpinga. Hartree-Fock Equation for a Non-neutral Plasma of Spin Zero Ions in a Paul Trap. American Journal of Physics and Applications. Vol. 4, No. 3, 2016, pp. 71-77. doi: 10.11648/j.ajpa.20160403.11

Received: April 11, 2016; Accepted: April 25, 2016; Published: May 11, 2016

\begin{abstract}
The imperceptible identical particle systems such as electron gas in metals have been the concern of experimental and theoretical studies mostly aiming to understand the properties of these systems. Hartree-Fock equation of electron gas, a fermion quantum plasma, has been established by the method of "equation of motion", and by using Dirac field. The goal of this paper is to establish regarding the same method, the Hartree-Fock equation for a non-neutral plasma of identical ions of spin zero at high density and low temperature in a Paul trap, by using the complex scalar field.
\end{abstract}

Keywords: Identical Ions, Spin Zero, Paul Trap, Non-neutral Plasma, Microscopic Theory, Field Operator, Fock Space, Hamiltonian Operator

\section{Introduction}

Cooled ions are used as quantum memories [1]. These identical ions comply with different statistics according to whether it is concerned with fermions or bosons. HartreeFock equation is an approximated equation of the dynamics of a system at low temperature [2] [3]. Identical ions are trapped in a Paul trap where they are cooled [1]. When the spin of these ions is zero, the dynamics of the system at high density and low temperature is given by the approximation equation which is the concern of this study. We use second quantization as the ions are identical [2] and microscopic theory because the interaction Coulombian in the boson plasma at high density is weak [3].

\section{Hartree-Fock Equation for a Non-neutral Plasma of Spin Zero Ions in a Paul Trap}

\subsection{Non-neutral Plasma in a Paul Trap}

In 2009, Sebastien Removille developed, in his Ph.D. dissertation, an experimental set capable of confining, cooling and observing several millions of ions. A linear Paul trap of centimetric dimensions has been drawn and made in a laboratory to confine ions and to facilitate a very low temperature system. Figure 1 shows a principle of linear Paul trap composed of six electrodes electrically connected in couples to make three pairs. Two pairs of cylindric electrodes insure the confinement of the cross movement $(\mathrm{X}, \mathrm{Y})$ and one pair of electrodes in rings insures the confinement of lengthwise movement (endcaps). Cylindrical bars are parallely set out and diagonally paired. They are referred to as electrodes pair RF and electrodes pair DC.

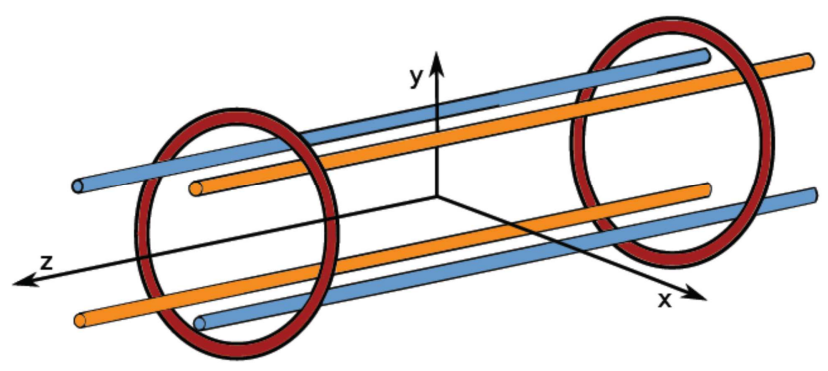

Source: Sebastien removille's thesis, 2009

Fig. 1. Linear Paul trap principle.

The usual constraints with a drawing of a trap are the precision of the parts, that of their position and the pertubations of the electrical field created by the environment of the electrodes, notably the fixing structure. These traps are 
used in ultra-vacuum chambers; which requires a choice of suitable materials. There is a tendancy within the community of ions trappers to use more and more small traps supplied with the very high frequency oscillating tension (voltage). On top of the integrability of the traps, the issue at stake in this tendancy is also to obtain a very important potential stiffness and to put ions at the weakest level of vibration [1].

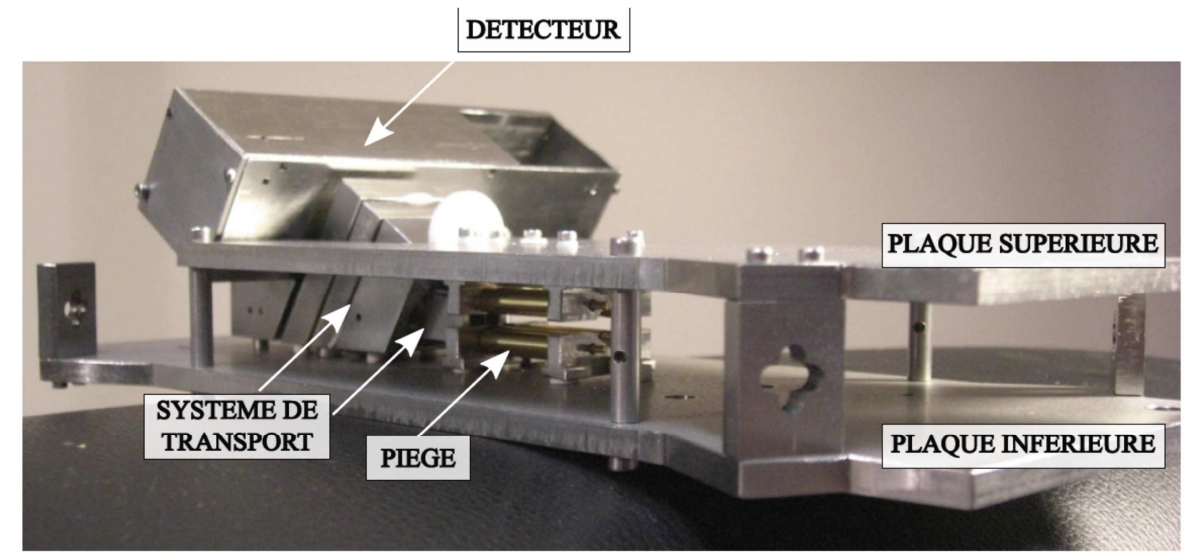

Source: Sebustein Removille's thesis, 2009

Fig. 2. Picture of the trapping and counting system of 2.0 type.

Figure 3 shows a cloud containing a thousand ions of strontium $\left(\mathrm{Sr}^{+}\right)$, the time of rest is of 5 seconds.

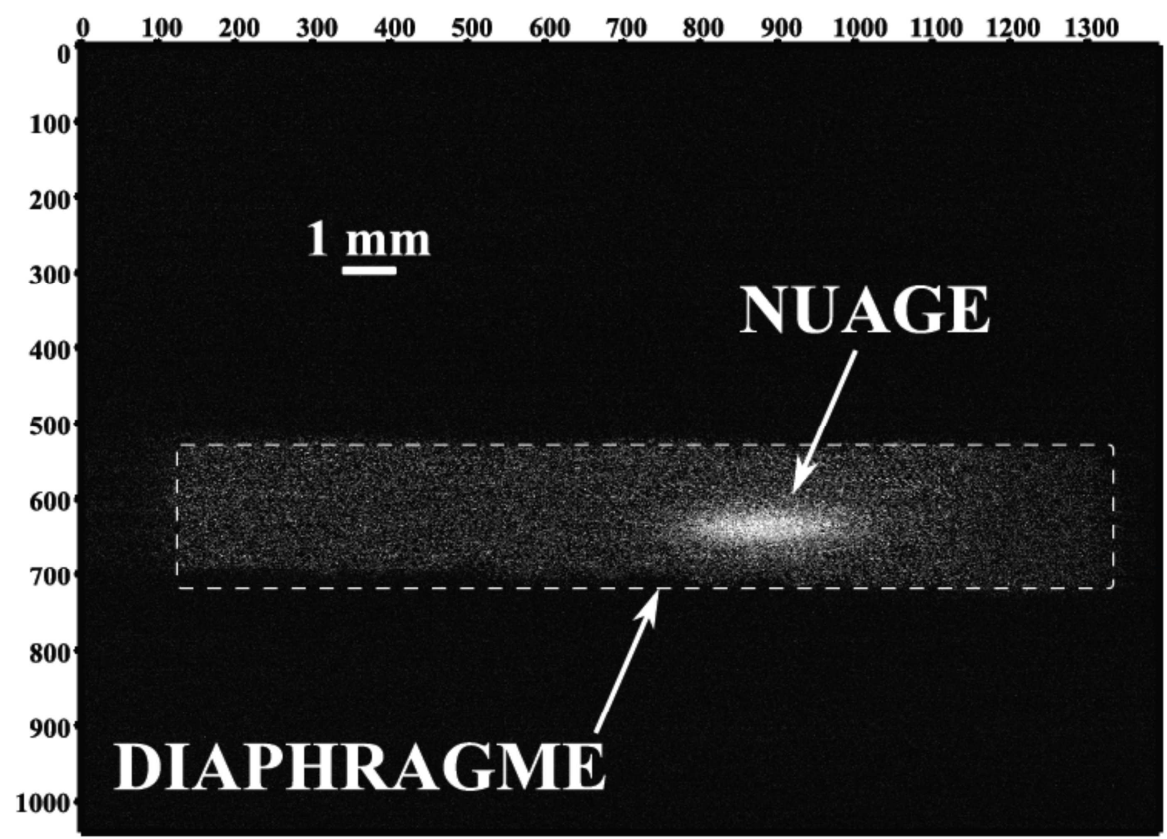

Source: Sebastien Removille's thesis, 2009.

Fig. 3. A negative of a cloud containing a thousand ions.

\subsection{Laser Cooling Technique}

The observation of correlated plasma has always been the researchers' inquiry into the ultra-cold plasmas. One can even envisage the quantum degeneration states by approaching Fermi's temperatures, theoretical predictions by Dresde's team demonstrated for instance, that by ionizing a perfectly ordered atomic gas, as coming from Mott's transition, it is possible to reach correlated atomic system. The same team also predicted that a laser cooling of plasma ions allows to execute an optical molass slowing down the expansion of plasma and allowed to reach a correlated ionic system [4].

For reaching a low temperature system, Sebastien used the laser cooling. This consists in lightening a cluster of ions by a laser practically resonant with an atomic transition. When the disagreement is negative, Doppler effect brings the resonant the ions whose speed is opposed to the sense of propagation of laser. Then, these ions preferentially absorb the photons whose quantity of movement is oppose to their speed, and are 
slow down. In the Paul trap, the ions being confined by the electrostatic trap, it is not necessary to create an optical molass. The effects of one single beam reduce the speed of ions in one sense of space direction, but these ions being confined, their total kinetic energy is reduced [1].

\subsection{Microscopic Theory}

The microscopic theory allows to study the properties of the groups of identical particles enclosed in a box or in a trap at high density or low temperature [3]. The quantum gas being made of indiscernable and identical particles, the use of the second quantization method offers a more flexible representation that is more concised and neater. The second quantization usually considers a group of particles more or less independent paired by weak interractions [2]. Particle operators which are concerned with second quantization are attributable to the quantum theory of fields [5]. The application of microscopic theory to the bosons in interaction is but satisfafied when there exist a weak repulsive interaction between particles or a gas diluated with arbitrary repulsive interaction between particles [3].

The microscopic theory is applied to a gathering of identical ions, at high density, confined in a trap because the Coulombian interaction is weak [6]. For electron systems there exist two such models; the high-density electron gas, and the low-density electron solid. The behavior of the electron liquid in the limit of very high densities is simple because here the Coulomb interaction represents a relatively small perturbation; the average potential energy is small compared to the kinetic energy. Where the inter-electron properties are well described in the so-called random phase approximation (RPA). In the low-density limit, the electron behavior is dominated by the Coulomb interaction. The region of actual metallic densities is essentially an intermediate coupling regime. As such it is for more difficult to treat; the kinetic energy and potential energy are comparable, and consequently no rigorous approximation schemas are applicable. One learns much about the behavior of electron metals by considering the RPA, despite the fact that it is valid only in the high-density limit [3].

The points of microscopic theory are:

- Particle field: in quantum theory of fields, particles are described by means of fields. These fields stand for KleinGordon solutions deriving from a free Lagrangian density which is an invariant of Lorentz admitting a positive Hamiltonian density [7]. For instance, electrons are described by means of Dirac field [2], the neutral bosons of spin zero by means of real scalar field [3], photons by means of real vectorial field [8], 1 spin charged bosons by means of complex vectorial field [9], spin zero charged bosons by means of complex scalar field or charged meson field [10];

- Particule operator (boson operator or fermion operator): once the particle field is known, it can be transformed into an operator; by inverse transform, the field coefficients become particle operators which satisfy at equal times commutation or anti-commutation relations [3];

- Space of system quantum states: any state of a system of identical particles can be described as a linear combination of many-particle basis states, a basis state can be completely specified in terms of the occupation number $n_{\alpha}$ for each member of a complete set of orthonormal single-particle states, the set of occupation numbers contains all the information necessary to construct an appropriately symmetrized or antisymmetrized basis vector. For bosons, $n_{\alpha}$ must be a non-negative integer; for fermions, the Pauli exclusion principle restricts $n_{\alpha}$ to be either 0 or 1 [11]. The vector space spanned by the set of all basis states is called the Fock space. A feature of the Fock space is that the total number of particles is not a fixed parameter, but rather is a dynamical variable associated with a total number operator $\mathrm{N}$;

- Field operators of annihilation and creation of particle, which must satisfy at equal times anti-commutation relations for fermions and commutation relations for bosons [3];

- Bogoliubov prescription for a system of particles in the fundamental state with interaction;

- Density matrix to a particle, defining the correlation between one particle at point $\vec{r}$ and another at point $\vec{r}^{\prime}$. This quantity depends only on the distance $\left|\vec{r}^{\prime}-\vec{r}\right|$ between two space points and leads to the long range order, characteristic of the existence of a Bose-Einstein condensate or to its depletion ;

- Hamiltonian of the second quantification of particles system;

- Energy calculation of the fundamental state;

- Spectrum of elementary excitations;

- Temporal evolution of the Heisenberg field operator, allowing to establish, for fundamental state, the GrossPitaevskii equation in case of neutral gas bosons of short range interaction; or Hartree-Fock equation for a particles system with long range interaction.

\subsection{Complex Scalar Field and Its Quantization}

The expansion of complex scalar field is written as follow [10], [7]:

$$
\begin{gathered}
\phi\left(x^{\mu}\right)=\frac{1}{\sqrt{(2 \pi)^{3}}} \int \frac{d^{3} k}{\sqrt{2 k_{0}}}\left[a e^{i k x}+b^{*} e^{-i k x}\right], \\
\phi^{*}\left(x^{\mu}\right)=\frac{1}{\sqrt{(2 \pi)^{3}}} \int \frac{d^{3} k}{\sqrt{2 k_{0}}}\left[b e^{i k x}+a^{*} e^{-i k x}\right] .
\end{gathered}
$$

The canonical quantization of complex scalar field consists of considering the field $\phi\left(\mathrm{x}^{\mu}\right)$ and its canonical conjugated $\pi\left(x^{\mu}\right)$ defined [12] as

$$
\pi\left(x^{\mu}\right)=\frac{\partial \mathcal{L}}{\partial \frac{\partial \phi}{\partial t}}=\frac{\partial \phi^{*}\left(x^{\mu}\right)}{\partial t},
$$

and also their complex conjugated $\phi^{*}\left(x^{\mu}\right)$ and $\pi^{*}\left(x^{\mu}\right)$, as operators which satisfy the following relations of commutation at equal times [10]

$$
\begin{gathered}
{\left[\hat{\phi}(\vec{r}, t), \hat{\pi}\left(\vec{r}^{\prime}, t\right)\right]=\left[\hat{\phi}^{\dagger}(\vec{r}, t), \hat{\pi}^{\dagger}\left(\vec{r}^{\prime}, t\right)\right]=i \delta^{3}\left(\vec{r}-\vec{r}^{\prime}\right),} \\
{\left[\hat{\phi}^{\dagger}(\vec{r}, t), \hat{\pi}\left(\vec{r}^{\prime}, t\right)\right]=\left[\hat{\phi}(\vec{r}, t), \hat{\pi}^{\dagger}\left(\vec{r}^{\prime}, t\right)\right]=0,}
\end{gathered}
$$




$$
\begin{aligned}
{\left[\hat{\pi}(\vec{r}, t), \hat{\pi}\left(\vec{r}^{\prime}, t\right)\right] } & =\left[\hat{\pi}^{\dagger}(\vec{r}, t), \hat{\pi}^{\dagger}\left(\vec{r}^{\prime}, t\right)\right] \\
& =\left[\hat{\pi}(\vec{r}, t), \hat{\pi}^{\dagger}\left(\vec{r}^{\prime}, t\right)\right]=0, \\
{\left[\hat{\phi}(\vec{r}, t), \hat{\phi}\left(\vec{r}^{\prime}, t\right)\right] } & =\left[\hat{\phi}^{\dagger}(\vec{r}, t), \hat{\phi}^{\dagger}\left(\vec{r}^{\prime}, t\right)\right] \\
& =\left[\hat{\phi}(\vec{r}, t), \hat{\phi}^{\dagger}\left(\vec{r}^{\prime}, t\right)\right]=0 .
\end{aligned}
$$

\subsection{Boson Operators and Commutation Relations}

By boson operators, we mean the operators $\hat{\mathrm{a}}(\overrightarrow{\mathrm{k}})$ and $\hat{\mathrm{b}}^{\dagger}(\overrightarrow{\mathrm{k}})$ and their adjuncts $\hat{a}^{\dagger}(\vec{k})$ and $\hat{b}(\vec{k})$ built from the coefficients of the solutions (1) and (2) of Klein Cordon equation. By applying the inverse Fourier transform of these solutions bellow [7], we obtain

$$
\begin{aligned}
& \mathrm{a}(\mathrm{k})=\frac{1}{\sqrt{(2 \pi)^{3}}} \int \frac{\mathrm{d}^{3} \mathrm{x}}{\sqrt{2 \mathrm{k}_{0}}} \mathrm{e}^{\mathrm{i} \mathrm{kx}} \stackrel{\leftrightarrow}{\partial_{0}} \phi\left(\mathrm{x}^{\mu}\right), \\
& b(k)=\frac{1}{\sqrt{(2 \pi)^{3}}} \int \frac{d^{3} x}{\sqrt{2 \mathrm{k}_{0}}} e^{\mathrm{ikx}} \stackrel{\leftrightarrow}{\partial_{0}} \phi^{*}\left(\mathrm{x}^{\mu}\right)
\end{aligned}
$$

This leads to

$$
\begin{aligned}
& \mathrm{a}(\mathrm{k})=\frac{1}{\sqrt{(2 \pi)^{3}}} \int \frac{\mathrm{d}^{3} \mathrm{x}}{\sqrt{2 \mathrm{k}_{0}}}\left[\frac{\partial \phi\left(\mathrm{x}^{\mu}\right)}{\partial \mathrm{t}}-\mathrm{ik}_{0} \phi\left(\mathrm{x}^{\mu}\right)\right] \mathrm{e}^{\mathrm{ikx}}, \\
& \mathrm{b}(\mathrm{k})=\frac{1}{\sqrt{(2 \pi)^{3}}} \int \frac{\mathrm{d}^{3} \mathrm{x}}{\sqrt{2 \mathrm{k}_{0}}}\left[\frac{\partial \phi^{*}\left(\mathrm{x}^{\mu}\right)}{\partial \mathrm{t}}-\mathrm{ik}_{0} \phi^{*}\left(\mathrm{x}^{\mu}\right)\right] \mathrm{e}^{\mathrm{ikx}} .
\end{aligned}
$$

The Fourier amplitudes of the field a (k) and b (k) as well as their complex conjugated are interpreted as destruction and creation operators which satisfy the following relations of commutation at equal time [7]

$$
\begin{aligned}
{\left[\hat{a}(k), \hat{a}^{\dagger}\left(k^{\prime}\right)\right]=} & {\left[\hat{b}(k), \hat{b}^{\dagger}\left(k^{\prime}\right)\right]=\delta^{(3)}\left(k-k^{\prime}\right), } \\
{\left[\hat{a}(k), \hat{a}\left(k^{\prime}\right)\right] } & =\left[\hat{b}(k), \hat{b}\left(k^{\prime}\right)\right]=\left[\hat{a}^{\dagger}(k), \hat{a}^{\dagger}\left(k^{\prime}\right)\right] \\
& =\left[\hat{b}^{\dagger}(k), \hat{b}^{\dagger}\left(k^{\prime}\right)\right]=\cdots=0 .
\end{aligned}
$$

$\hat{a}^{\dagger}\left(k^{\prime}\right)$ and $\hat{b}^{\dagger}\left(k^{\prime}\right)$ are the creation operators of particle whereas $\hat{a}(k)$ and $\hat{b}(k)$ are the destruction operators of particle.

\subsection{Space of Quantum States of Boson Operators}

The space of states of boson systems is built by acting on the state of the Fock vacuum with the boson operators under the following conditions [10] [6] [13]:

$$
\hat{a}(k)|0>=0=\hat{b}(k)| 0>,\langle 0 \mid 0\rangle=1
$$

The Fock base of the space quantum states of the system is given by

$$
\left|n_{1}, n_{2}\right\rangle=\frac{1}{\sqrt{n_{1} ! n_{2} !}}\left(\hat{a}^{\dagger}\right)^{n_{1}}\left(\hat{b}^{\dagger}\right)^{n_{2}}|0\rangle,
$$

and

$$
\left\langle n_{1}, n_{2} \mid m_{1}, m_{2}\right\rangle=\delta_{n_{1}, m_{1}} \delta_{n_{2}, m_{2}} .
$$

\subsection{Field Operators of Destruction or Creation of Particle of Impulsion p at the Point $r$}

The building of the destruction field operators is simply done from $\hat{a}_{p}$ and $\hat{b}_{p}$. The development of $|\vec{r}\rangle$ is used on the base $\{|\vec{p}\rangle\}[3][13]$

$$
\langle\vec{r} \mid \vec{p}\rangle=\frac{1}{\sqrt{\Omega}} e^{i \vec{k} \cdot \vec{r}}=\varphi_{k}(r) .
$$

We obtain the field operator of destruction of boson and anti-boson by [6]

$$
\hat{\psi}(r)=\frac{1}{\sqrt{\Omega}} \sum_{k}\left(\hat{a}_{k}+\hat{b}_{k}\right) e^{i \vec{k} \cdot \vec{r}}=\sum_{k}\left(\hat{a}_{k}+\hat{b}_{k}\right) \varphi_{k}(r) .
$$

The field operator of creation of boson and anti-boson is obtained by

$$
\begin{aligned}
\hat{\psi}^{\dagger}(r) & =\sum_{k}\left(\hat{a}_{k}^{\dagger}+{\hat{b}_{k}}^{\dagger}\right)\langle\vec{p} \mid \vec{r}\rangle \\
& =\frac{1}{\sqrt{\Omega}} \sum_{k}\left({\widehat{a}_{k}}^{\dagger}+\hat{b}_{k}^{\dagger}\right) e^{-i \vec{k} \cdot \vec{r}} \\
& =\sum_{k}\left(\hat{a}_{k}^{\dagger}+\hat{b}_{k}^{\dagger}\right) \varphi_{k}^{*}(r) .
\end{aligned}
$$

These operators, with respect to Eqs. (10), (11) and (15), satisfy the following relations of commutation at equal times [6]

$$
\begin{aligned}
& {\left[\hat{\psi}(r), \hat{\psi}^{\dagger}\left(r^{\prime}\right)\right]_{-} }=\left[\sum_{k}\left(\hat{a}_{k}+\hat{b}_{k}\right) \varphi_{k}(r), \sum_{k}\left(\hat{a}_{k}^{\dagger}+\hat{b}_{k}^{\dagger}\right) \varphi_{k}^{*}\left(r^{\prime}\right)\right] \\
&=2 \delta\left(r-r^{\prime}\right), \\
& {\left[\hat{\psi}(r), \hat{\psi}\left(r^{\prime}\right)\right]_{-}=\left[\hat{\psi}^{\dagger}(r), \hat{\psi}^{\dagger}\left(r^{\prime}\right)\right]_{-}=0 . }
\end{aligned}
$$

\subsection{Hamiltonian Operator of Non- Neutral Plasma}

The Hamiltonian operator of the boson system in interaction is defined in second quantization, and in Schrödinger picture by [3] [5] [14]

$$
\begin{gathered}
\widehat{H}=\int d^{3} r \hat{\psi}^{\dagger}(r)\left[\frac{\hat{p}^{2}}{2 m}+\hat{V}_{p i e ̀ g e}\right] \widehat{\psi}(r) \\
+\frac{1}{2} \iint d^{3} r d^{3} r^{\prime} \hat{\psi}^{\dagger}(r) \hat{\psi}^{\dagger}\left(r^{\prime}\right) \hat{V}\left(r-r^{\prime}\right) \hat{\psi}\left(r^{\prime}\right) \hat{\psi}(r) .
\end{gathered}
$$

\subsection{Temporal Evolution of the Field Operator of Zero Spin Ion}

The temporal evolution of the field operator $\widehat{\psi}(\vec{r}, \mathrm{t})$ is defined [2] [3]

$$
i \hbar \frac{\partial \widehat{\psi}(\vec{r}, t)}{\partial t}=[\widehat{\psi}(\vec{r}, t), \widehat{H}] .
$$

Equation (21) can be written [15]

$$
i \frac{\partial \widehat{\psi}(\vec{r}, t)}{\partial t}=-[\widehat{H}, \widehat{\psi}(\vec{r}, t)], \text { where } \hbar \equiv 1 .
$$

Let's calculate the commutation of this Eq. (22) with respect to the relations (20) in Heisenberg picture, (18) and (19). We use the notations $\widehat{\psi}\left(\mathrm{r}^{\prime \prime}\right) \equiv \widehat{\psi}\left(\vec{r}^{\prime \prime}, t\right), \widehat{\psi}\left(\mathrm{r}^{\prime}\right) \equiv$ $\widehat{\psi}\left(\vec{r}^{\prime}, \mathrm{t}\right)$ and $\widehat{\psi}(\mathrm{r}) \equiv \widehat{\psi}(\vec{r}, \mathrm{t})$ 
$\left[\widehat{H}, \hat{\psi}\left(r^{\prime \prime}\right)\right]=\int d^{3} r\left[\hat{\psi}^{\dagger}(r)\left(\frac{\hat{p}^{2}}{2 m}+\widehat{V}_{p i \grave{g} g e}\right) \hat{\psi}(r), \widehat{\psi}\left(r^{\prime \prime}\right)\right]$ $+\frac{1}{2} \iint d^{3} r d^{3} r^{\prime}\left[\hat{\psi}^{\dagger}(r) \hat{\psi}^{\dagger}\left(r^{\prime}\right) \hat{V}\left(r-r^{\prime}\right) \hat{\psi}\left(r^{\prime}\right) \hat{\psi}(r), \hat{\psi}\left(r^{\prime \prime}\right)\right] .(23)$

The first term on the right is worth

$$
\begin{aligned}
& \int d^{3} r\left[\hat{\psi}^{\dagger}(r)\left(\frac{\hat{p}^{2}}{2 m}+\hat{V}_{\text {piège }}\right) \hat{\psi}(r), \hat{\psi}\left(r^{\prime \prime}\right)\right] \\
& =\int d^{3} r \hat{\psi}^{\dagger}(r)\left(\frac{\hat{p}^{2}}{2 m}\right. \\
& \left.+\widehat{V}_{\text {piège }}\right)\left[\hat{\psi}(r), \hat{\psi}\left(r^{\prime \prime}\right)\right] \\
& +\int d^{3} r\left[\hat{\psi}^{\dagger}(r), \hat{\psi}\left(r^{\prime \prime}\right)\right]\left(\frac{\hat{p}^{2}}{2 m}+\hat{V}_{\text {piège }}\right) \hat{\psi}(r) \\
& =0+\int d^{3} r(-) \delta\left(r^{\prime \prime}-r\right)\left(\frac{\hat{p}^{2}}{2 m}+\widehat{V}_{\text {piège }}\right) \hat{\psi}(r) \\
& =-\left(\frac{\hat{p}^{2}}{2 m}+\widehat{V}_{p i e ̀ g e}\right) \hat{\psi}\left(r^{\prime \prime}\right) .
\end{aligned}
$$

The calculation of the second term on the right of Eq. (23) gives

$$
\begin{gathered}
\frac{1}{2} \iint d^{3} r d^{3} r^{\prime}\left[\hat{\psi}^{\dagger}(r) \hat{\psi}^{\dagger}\left(r^{\prime}\right) \hat{V}\left(r-r^{\prime}\right) \hat{\psi}\left(r^{\prime}\right) \hat{\psi}(r), \hat{\psi}\left(r^{\prime \prime}\right)\right] \\
=\frac{1}{2} \iint d^{3} r d^{3} r^{\prime} \hat{\psi}^{\dagger}(r)\left[\hat{\psi}^{\dagger}\left(r^{\prime}\right), \hat{\psi}\left(r^{\prime \prime}\right)\right] \hat{V}\left(r-r^{\prime}\right) \hat{\psi}\left(r^{\prime}\right) \hat{\psi}(r) \\
+\frac{1}{2} \iint d^{3} r d^{3} r^{\prime}\left[\hat{\psi}^{\dagger}(r), \hat{\psi}\left(r^{\prime \prime}\right)\right] \hat{\psi}^{\dagger}\left(r^{\prime}\right) \hat{V}\left(r-r^{\prime}\right) \hat{\psi}\left(r^{\prime}\right) \hat{\psi}(r) \\
=\frac{1}{2} \iint d^{3} r d^{3} r^{\prime} \hat{\psi}^{\dagger}(r)(-) \delta\left(r^{\prime \prime}-r^{\prime}\right) \hat{V}\left(r-r^{\prime}\right) \hat{\psi}\left(r^{\prime}\right) \hat{\psi}(r) \\
+\frac{1}{2} \iint d^{3} r d^{3} r^{\prime}(-) \delta\left(r^{\prime \prime}-r\right) \hat{\psi}^{\dagger}\left(r^{\prime}\right) \hat{V}\left(r-r^{\prime}\right) \hat{\psi}\left(r^{\prime}\right) \hat{\psi}(r) \\
=-\frac{1}{2} \int d^{3} r \hat{\psi}^{\dagger}(r)\left(\int d^{3} r^{\prime} \delta\left(r^{\prime \prime}-r^{\prime}\right) \hat{V}\left(r-r^{\prime}\right) \hat{\psi}\left(r^{\prime}\right)\right) \hat{\psi}(r) \\
-\frac{1}{2} \int d^{3} r^{\prime} \hat{\psi}^{\dagger}\left(r^{\prime}\right) \hat{\psi}\left(r^{\prime}\right)\left(\int d^{3} r \delta\left(r^{\prime \prime}-r\right) \hat{V}\left(r-r^{\prime}\right) \hat{\psi}(r)\right) \\
=-\frac{1}{2} \int d^{3} r \hat{V}\left(r^{\prime \prime}-r^{\prime}\right) \hat{\psi}^{\dagger}(r) \hat{\psi}\left(r^{\prime \prime}\right) \hat{\psi}(r) \\
-\frac{1}{2} \int d^{3} r^{\prime} \hat{V}\left(r^{\prime \prime}-r^{\prime}\right) \hat{\psi}^{\dagger}\left(r^{\prime}\right) \hat{\psi}\left(r^{\prime}\right) \hat{\psi}\left(r^{\prime \prime}\right)
\end{gathered}
$$

The potential $\widehat{V}\left(\mathrm{r}^{\prime \prime}-\mathrm{r}^{\prime}\right)$ concerns the points $r^{\prime \prime}$ and $r^{\prime}$, hence

$$
\begin{gathered}
\frac{1}{2} \iint d^{3} r d^{3} r^{\prime}\left[\widehat{\psi}^{\dagger}(r) \widehat{\Psi}^{\dagger}\left(r^{\prime}\right) \widehat{V}\left(r-r^{\prime}\right) \widehat{\psi}\left(r^{\prime}\right) \widehat{\psi}(r), \widehat{\psi}\left(r^{\prime \prime}\right)\right]= \\
-\int d^{3} r^{\prime} \widehat{V}\left(r^{\prime \prime}-r^{\prime}\right) \widehat{\Psi}^{\dagger}\left(r^{\prime}\right) \widehat{\psi}\left(r^{\prime}\right) \widehat{\psi}\left(r^{\prime \prime}\right) .
\end{gathered}
$$

By including Eqs. (25) and (24) in Eq. (23), we obtain

$$
\left[\widehat{H}, \hat{\psi}\left(r^{\prime \prime}\right)\right]=-\frac{\left(\frac{\hat{p}^{2}}{2 m}+\hat{V}_{p i e ̀ g e}\right) \hat{\psi}\left(r^{\prime \prime}\right)-\int d^{3} r^{\prime} \hat{V}\left(r^{\prime \prime}-\right.}{\left.r^{\prime}\right) \hat{\psi}^{\dagger}\left(r^{\prime}\right) \hat{\psi}\left(r^{\prime}\right) \hat{\psi}\left(r^{\prime \prime}\right)}
$$

In Eq. (26), the second term on the right has the following form:

$$
\begin{gathered}
\int d^{3} r^{\prime} \hat{V}\left(r^{\prime \prime}-r^{\prime}\right) \hat{\psi}^{\dagger}\left(r^{\prime}\right) \hat{\psi}\left(r^{\prime}\right) \hat{\psi}\left(r^{\prime \prime}\right) \\
=\sum_{k l m} \hat{a}_{k}^{\dagger} \hat{a}_{l} \hat{a}_{m} \int d^{3} r^{\prime} \hat{V}\left(r^{\prime \prime}-r^{\prime}\right) \varphi_{k}^{*}\left(r^{\prime}\right) \varphi_{l}\left(r^{\prime}\right) \varphi_{m}\left(r^{\prime \prime}\right) .
\end{gathered}
$$

The member on the right of Eq. (27) contains the products of the three bosons operators. In the approximation of the first order called Hartree-Fock, we only consider the terms including the number operator $\hat{\mathrm{a}}_{\mathrm{k}}^{\dagger} \hat{\mathrm{a}}_{\mathrm{k}}$. We also preserve the terms $\hat{a}_{k}^{\dagger} \hat{a}_{k} \hat{a}_{m}$ and $\hat{a}_{k}^{\dagger} \hat{a}_{l} \hat{a}_{k}$. Eq. (27) becomes therefore [2] [3]

$$
\begin{aligned}
& \int d^{3} r^{\prime} \hat{V}\left(r^{\prime \prime}-r^{\prime}\right) \hat{\psi}^{\dagger}\left(r^{\prime}\right) \hat{\psi}\left(r^{\prime}\right) \hat{\psi}\left(r^{\prime \prime}\right) \\
& \cong \int d^{3} r^{\prime} \hat{V}\left(r^{\prime \prime}-r^{\prime}\right)\left\langle\hat{\psi}^{\dagger}\left(r^{\prime}\right) \hat{\psi}\left(r^{\prime}\right)\right\rangle \hat{\psi}\left(r^{\prime \prime}\right) \\
&+\int d^{3} r^{\prime} \widehat{V}\left(r^{\prime \prime}-r^{\prime}\right) \hat{\psi}\left(r^{\prime}\right)\left\langle\hat{\psi}^{\dagger}\left(r^{\prime}\right) \hat{\psi}\left(r^{\prime \prime}\right)\right\rangle .
\end{aligned}
$$

Where we have summed the series which appear in the expressions of $\widehat{\psi}\left(\mathrm{r}^{\prime \prime}\right)$ and $\widehat{\psi}\left(\mathrm{r}^{\prime}\right)$; the terms between brackets indicate the probable values in the fundamental state, that is, only the terms in $\hat{a}_{k}^{\dagger} \hat{a}_{k}$ are retained inside the brackets and $\hat{\mathrm{a}}_{\mathrm{k}}^{\dagger} \hat{\mathrm{a}}_{\mathrm{k}}$ is calculated for fundamental state. The first term on the right of Eq. (28) represents the direct Coulombian action, the second the exchange [2].

Equation (28) allows Eq. (26) to be become

$$
\begin{gathered}
{\left[\widehat{H}, \hat{\psi}\left(r^{\prime \prime}\right)\right] \cong\left(-\frac{\hat{p}^{2}}{2 m}-\widehat{V}_{\text {piège }}\right.} \\
-\int d^{3} r^{\prime} \widehat{V}\left(r^{\prime \prime}\right. \\
\left.\left.-r^{\prime}\right)\left\langle\hat{\psi}^{\dagger}\left(r^{\prime}\right) \hat{\psi}\left(r^{\prime}\right)\right\rangle\right) \hat{\psi}\left(r^{\prime \prime}\right) \\
-\int \mathrm{d}^{3} \mathrm{r}^{\prime} \widehat{V}\left(\mathrm{r}^{\prime \prime}-\mathrm{r}^{\prime}\right) \widehat{\psi}\left(\mathrm{r}^{\prime}\right)\left\langle\widehat{\psi}^{\dagger}\left(\mathrm{r}^{\prime}\right) \widehat{\psi}\left(\mathrm{r}^{\prime \prime}\right)\right\rangle .
\end{gathered}
$$

In including Eq. (29) into Eq. (22), we obtain the temporal evolution of Heisenberg field operator

$$
\begin{gathered}
i \frac{\partial \hat{\psi}\left(r^{\prime \prime}\right)}{\partial t}=-\left[\widehat{H}, \hat{\psi}\left(r^{\prime \prime}\right)\right] \\
\cong\left(\frac{\hat{p}^{2}}{2 m}+\widehat{V}_{p i \grave{g} g e}+\int d^{3} r^{\prime} \hat{V}\left(r^{\prime \prime}-r^{\prime}\right)\left\langle\hat{\psi}^{\dagger}\left(r^{\prime}\right) \hat{\psi}\left(r^{\prime}\right)\right\rangle\right) \hat{\psi}\left(r^{\prime \prime}\right) \\
+\int d^{3} r^{\prime} \hat{\psi}\left(r^{\prime}\right) \widehat{V}\left(r^{\prime \prime}-r^{\prime}\right)\left\langle\hat{\psi}^{\dagger}\left(r^{\prime}\right) \hat{\psi}\left(r^{\prime \prime}\right)\right\rangle .
\end{gathered}
$$

\subsection{Hartree- Fock Equation of Identical Ions of Spin Zero}

The Heisenberg field operator of spin zero charged particle, Eq. (16), may take the form [6] 


$$
\begin{aligned}
\hat{\psi}\left(\vec{r}^{\prime \prime}, t\right) \equiv \hat{\psi}\left(r^{\prime \prime}\right) & =\sum_{k} \hat{a}_{k} \varphi_{k}\left(r^{\prime \prime}\right)+\sum_{k} \hat{b}_{k} \varphi_{k}\left(r^{\prime \prime}\right) \\
& =\hat{\psi}_{+}\left(r^{\prime \prime}\right)+\hat{\psi}_{-}\left(r^{\prime \prime}\right) .
\end{aligned}
$$

Let's consider Eq. (30) in the case of positive charged particle (boson),

$$
\begin{aligned}
& i \frac{\partial \hat{\psi}_{+}\left(r^{\prime \prime}\right)}{\partial t} \cong\left(\frac{\hat{p}^{2}}{2 m}+\hat{V}_{\text {piège }}\right. \\
& +\int d^{3} r^{\prime} \widehat{V}\left(r^{\prime \prime}\right. \\
& \left.\left.-r^{\prime}\right)\left\langle\hat{\psi}_{+}^{\dagger}\left(r^{\prime}\right) \hat{\psi}_{+}\left(r^{\prime}\right)\right\rangle\right) \hat{\psi}_{+}\left(r^{\prime \prime}\right)
\end{aligned}
$$

$$
+\int d^{3} r^{\prime} \hat{\psi}_{+}\left(r^{\prime}\right) \widehat{V}\left(r^{\prime \prime}-r^{\prime}\right)\left\langle\hat{\psi}_{+}^{\dagger}\left(r^{\prime}\right) \hat{\psi}_{+}\left(r^{\prime \prime}\right)\right\rangle
$$

We well known, on the hand, that in Hilbert space

$$
\hat{A}\lceil\varphi\rangle=a\lceil\varphi\rangle,
$$

and

$$
\begin{aligned}
&\lceil\varphi\rangle \equiv \varphi\left(r^{\prime \prime}\right), \text { where }\left(r^{\prime \prime}\right) \equiv\left(\overrightarrow{r^{\prime \prime}}, t\right), \quad \text { (34) Equation. (39) allows Eq. (32) to take } \\
& \sum_{j} \hat{a}_{j} \varepsilon_{j} \varphi_{j}\left(r^{\prime \prime}\right) \cong\left(\frac{\hat{p}^{2}}{2 m}+\widehat{V}_{\text {piège }}+\int d^{3} r^{\prime} \widehat{V}\left(r^{\prime \prime}-r^{\prime}\right)\left\langle\hat{\psi}_{+}^{\dagger}\left(r^{\prime}\right) \hat{\psi}_{+}\left(r^{\prime}\right)\right\rangle\right) \hat{\psi}_{+}\left(r^{\prime \prime}\right) \\
&+\int d^{3} r^{\prime} \hat{\psi}_{+}\left(r^{\prime}\right) \widehat{V}\left(r^{\prime \prime}-r^{\prime}\right)\left\langle\hat{\psi}_{+}^{\dagger}\left(r^{\prime}\right) \hat{\psi}_{+}\left(r^{\prime \prime}\right)\right\rangle,
\end{aligned}
$$

on the other hand

$$
\widehat{H} \varphi_{j}\left(r^{\prime \prime}\right)=\varepsilon_{j} \varphi_{j}\left(r^{\prime \prime}\right),
$$

or

$$
i \hbar \frac{d}{d t} \varphi_{j}\left(r^{\prime \prime}\right)=\varepsilon_{j} \varphi_{j}\left(r^{\prime \prime}\right) .
$$

Equation (36) may take the form

$$
i \hbar \frac{d}{d t} \sum_{j} \hat{a}_{j} \varphi_{j}\left(r^{\prime \prime}\right)=\sum_{j} \hat{a}_{j} \varepsilon_{j} \varphi_{j}\left(r^{\prime \prime}\right)
$$

or

$$
i \hbar \frac{d}{d t} \widehat{\psi}_{+}\left(r^{\prime \prime}\right)=\sum_{j} \hat{a}_{j} \varepsilon_{j} \varphi_{j}\left(r^{\prime \prime}\right) .
$$

From Eq. (21), the temporal evolution of $\hat{\psi}_{+}\left(r^{\prime \prime}\right)$ may take the form

$$
i \hbar \frac{d}{d t} \widehat{\psi}_{+}\left(r^{\prime \prime}\right)=\left[\widehat{\psi}_{+}\left(r^{\prime \prime}\right), \widehat{H}\right]=-\left[\widehat{H}, \widehat{\psi}_{+}\left(r^{\prime \prime}\right)\right] .
$$

Taking into account Eq. (37) we obtain

$$
\left[\widehat{H}, \hat{\psi}_{+}\left(r^{\prime \prime}\right)\right]=-\sum_{j} \hat{a}_{j} \varepsilon_{j} \varphi_{j}\left(r^{\prime \prime}\right) .
$$

Equation. (39) allows Eq. (32) to take the form

and

$$
\sum_{j} \hat{a}_{j} \varepsilon_{j} \varphi_{j}\left(r^{\prime \prime}\right) \cong \sum_{j} \hat{a}_{j}\left[\begin{array}{c}
\left(\frac{\hat{p}^{2}}{2 m}+\hat{V}_{p i e ̀ g e}+\int d^{3} r^{\prime} \hat{V}\left(r^{\prime \prime}-r^{\prime}\right)\left\langle\hat{\psi}_{+}^{\dagger}\left(r^{\prime}\right) \hat{\psi}_{+}\left(r^{\prime}\right)\right\rangle\right) \varphi_{j}\left(r^{\prime \prime}\right) \\
+\int d^{3} r^{\prime} \varphi_{j}\left(r^{\prime}\right) \widehat{V}\left(r^{\prime \prime}-r^{\prime}\right)\left\langle{\psi_{+}}^{\dagger}\left(r^{\prime}\right) \hat{\psi}_{+}\left(r^{\prime \prime}\right)\right\rangle
\end{array}\right]
$$

We suppose now that $\varphi_{j}\left(r^{\prime \prime}\right)$ are proper functions of the operator between the hook on the right of Eq. (40) and correspond to proper values $\varepsilon_{j},[12]$ then we have

$$
\begin{aligned}
\varepsilon_{j} \varphi_{j}\left(r^{\prime \prime}\right) & =\left(\frac{\hat{p}^{2}}{2 m}+\widehat{V}_{p i e ̀ g e}+\int d^{3} r^{\prime} \hat{V}\left(r^{\prime \prime}-r^{\prime}\right)\left\langle\hat{\psi}_{+}^{\dagger}\left(r^{\prime}\right) \hat{\psi}_{+}\left(r^{\prime}\right)\right\rangle\right) \varphi_{j}\left(r^{\prime \prime}\right) \\
& +\int d^{3} r^{\prime} \varphi_{j}\left(r^{\prime}\right) \widehat{V}\left(r^{\prime \prime}-r^{\prime}\right)\left\langle\hat{\psi}_{+}^{\dagger}\left(r^{\prime}\right) \hat{\psi}_{+}\left(r^{\prime \prime}\right)\right\rangle,
\end{aligned}
$$

hence

$$
\begin{aligned}
\varepsilon_{j} \varphi_{j}\left(r^{\prime \prime}\right)= & \left(\frac{\hat{p}^{2}}{2 m}+\hat{V}_{p i \grave{e} g e}+\int d \vec{r}^{\prime} \widehat{V}\left(r^{\prime \prime}-r^{\prime}\right) \sum_{\vec{l}} n_{i}^{(+)} \varphi_{i}^{*}\left(r^{\prime}\right) \varphi_{i}\left(r^{\prime}\right)\right) \varphi_{j}\left(r^{\prime \prime}\right) \\
& +\int d \vec{r}^{\prime} \varphi_{j}\left(r^{\prime}\right) \widehat{V}\left(r^{\prime \prime}-r^{\prime}\right) \sum_{i} n_{i}^{(+)} \varphi_{i}^{*}\left(r^{\prime}\right) \varphi_{i}\left(r^{\prime \prime}\right) .
\end{aligned}
$$

Equation (42) is the Hartree-Fock equation of spin zero positive boson in the Paul trap. By the same reasoning we obtain for spin zero negative boson in the Paul trap, the equation 


$$
\begin{aligned}
\varepsilon_{j} \varphi_{j}\left(r^{\prime \prime}\right) & =\left(\frac{\hat{p}^{2}}{2 m}+\widehat{V}_{\text {piège }}+\int d \vec{r}^{\prime} \widehat{V}\left(r^{\prime \prime}-r^{\prime}\right) \sum_{\vec{i}} n_{i}^{(-)} \varphi_{i}^{*}\left(r^{\prime}\right) \varphi_{i}\left(r^{\prime}\right)\right) \varphi_{j}\left(r^{\prime \prime}\right) \\
& +\int d \vec{r}^{\prime} \varphi_{j}\left(r^{\prime}\right) \hat{V}\left(r^{\prime \prime}-r^{\prime}\right) \sum_{i} n_{i}^{(-)} \varphi_{i}^{*}\left(r^{\prime}\right) \varphi_{i}\left(r^{\prime \prime}\right) .
\end{aligned}
$$

\section{Conclusion}

The Dirac field was used to determine the Hartree-Fock equation for electron gas which is a fermion quantum plasma. We use for identical ions with spin zero the charged mesons field. In equations (42) and (43), the exchanges (second term on the right) are added to direct Coulombian actions unlike the case of fermions.

\section{References}

[1] S. Removille., Vers une mémoire quantique dans des ions piégés. Thèse de doctorat, Université Paris. Diderot (Paris 7) 2009.

[2] C. Kittel. Théorie Quantique du Solide. (Dunod, Paris, 1967).

[3] P. Nozières and D. Pines. The theory of quantum liquids. 3th edition (Perseus books, Cambridge, 1999).

[4] D. Comparat (2008). Expériences avec des atomes de Rydberg et des molécules ultrafroids. Mémoire d'Habilitation à Diriger des Recherches.

[5] B.M. Caradoc-Davies. Vortex Dynamics in Bose-Einstein Condensates, These, University of Otago, 200 pages (2000).

[6] F. M Tshizanga, P. M. Badibanga and B. B. Ntampaka (2014), An investigation into the Parameters of Quantum Degeneration of an Ultra Cold Non-Neutre Plasma of identical Ions of Zero Spin in a Paul Trap. International Journal of Measurement Technologies and Instrumentation Engineering, 4(1), 51-70. January-March 2014

[7] F. Mandl, Introduction to Quantum Field Theory. 2th edition (Interscience Publishers INC., New York. 1964)

[8] S. Froit (2006), Introduction à la théorie des champs phénoménologique, INRIA.

[9] Govaerts, J. (1995)., L'interaction électrofaible: une fenêtre sur la physique au-delà du Modèle Standard, Paru dans les Actes de l'Ecole Internationale Joliot-Curie de Physique Nucléaire", Maubuisson (France) (pp. 333-416).

[10] S. Weinberg. The quantum theory of fields. Volume 15 th edition (Cambridge university press, volume I and II. New York, 2000)

[11] K. Ingersent, (2003), Second Quantization: Creation and Annihilation Operators, PHY 6646.

[12] A. S. Davydov. Quantum mechanics. (Pergamon Press, New York, 1965)

[13] J-P. Derendinger. Théorie quantique des champs. (Presses polytechniques et universitaires romandes, 2001)

[14] F. Dalfovo, S. Giorgini, L. P. Pitaevskii and S. Stringari (1999), Theory of Bose-Einstein condensation in trapped gases. Reviews of Modern Physics, Vol. 71, No. 3, April, pp 463-512

[15] J. S. Bell, Théorie Quantique des Champs Expérimentale. (CERN 77-18, Paris, 1977). 